\title{
IMA Commission on New Minerals, Nomenclature and Classification (CNMNC) - Newsletter 52
}

\author{
Ritsuro Miyawaki ${ }^{1}$, Frédéric Hatert $^{2}$, Marco Pasero ${ }^{3}$, and Stuart J. Mills ${ }^{4}$ \\ ${ }^{1}$ Chairman, CNMNC I Department of Geology, National Museum of Nature and Science, \\ 4-1-1 Amakubo, Tsukuba 305-0005, Japan \\ ${ }^{2}$ Vice-Chairman, CNMNC I Laboratoire de Minéralogie, Université de Liège, Bâtiment B18, \\ Sart Tilman, 4000 Liège, Belgium \\ ${ }^{3}$ Vice-Chairman, CNMNC I Dipartimento di Scienze della Terra, Università di Pisa, \\ Via Santa Maria 53, 56126 Pisa, Italy \\ ${ }^{4}$ Secretary, CNMNC I Geosciences, Museum Victoria, P.O. Box 666, Melbourne, Victoria 3001, Australia \\ Correspondence: Marco Pasero (marco.pasero@unipi.it) \\ Published: 14 January 2020
}

The information given here is provided by the IMA Commission on New Minerals, Nomenclature and Classification for comparative purposes and as a service to mineralogists working on new species.

Each mineral is described in the following format:

- mineral name, if the authors agree on its release prior to the full description appearing in press;

- chemical formula;

- type locality;

- full authorship of proposal;

- e-mail address of corresponding author;

- relationship to other minerals;

- crystal system, space group; structure determined, yes or no;

- unit-cell parameters;

- strongest lines in the X-ray powder diffraction pattern;

- type specimen repository and specimen number;

- citation details for the mineral prior to publication of full description.

Citation details concern the fact that this information will be published in the European Journal of Mineralogy on a routine basis, as well as being added month by month to the commission's web site.

It is still a requirement for the authors to publish a full description of the new mineral.

No other information will be released by the commission.

1 New mineral proposals approved in October 2019

IMA no. 2018-112

Esdanaite-(Ce)

$\mathrm{NaMnCe}\left(\mathrm{PO}_{4}\right)_{2} \cdot 4 \mathrm{H}_{2} \mathrm{O}$

Poudrette Quarry, Mont Saint-Hilaire, La Vallée-duRichelieu, Montérégie, Québec, Canada (453' $46^{\prime \prime} \mathrm{N}$, $73^{\circ} 8^{\prime} 30^{\prime \prime} \mathrm{W}$ )

Thomas E. Gore* and Andrew M. McDonald

*E-mail: tgore@laurentian.ca

New structure type

Orthorhombic: $P 2{ }_{1} 2_{1} 2_{1}$; structure determined

$a=6.469(1), b=7.169(1), c=21.456(4) \AA$

10.997(100), 3.605(28), 3.236(28), 2.951(21),

$2.778(29), 2.162(18), 2.005(16), 1.908(17)$ 
Type material is deposited in the mineralogical collections of the Canadian Museum of Nature, P.O. Box 3443, Station D, Ottawa, Ontario K1P 6P4, Canada, catalogue number CNMNC 87259.

How to cite: Gore, T. E. and McDonald, A. M.: Esdanaite-(Ce), IMA 2018-112, in: CNMNC Newsletter 52, Eur. J. Mineral, 32, https://doi.org/10.5194/ejm32-1-2020, 2020.

\section{IMA no. 2018-168}

Melansonite

$\mathrm{Na} \square \mathrm{KZrSi}_{8} \mathrm{O}_{19} \cdot 5 \mathrm{H}_{2} \mathrm{O}$

Poudrette Quarry, Mont Saint-Hilaire, La Valléedu-Richelieu RCM, Montérégie, Québec, Canada $\left(45^{\circ} 33^{\prime} 46^{\prime \prime} \mathrm{N}, 73^{\circ} 8^{\prime} 30^{\prime \prime} \mathrm{W}\right)$

Thomas E. Gore* and Andrew M. McDonald

*E-mail: tgore@laurentian.ca

Rhodesite group

Orthorhombic: Pmma; structure determined

$a=24.063(5), b=6.982(1), c=6.526(1) \AA$

11.988(100), $\quad 6.971(27), \quad 6.529(19), \quad 4.429(15)$, $3.429(15), 3.060(29), 2.881(25), 2.750(11)$

Type material is deposited in the mineralogical collections of the Canadian Museum of Nature, P.O. Box 3443, Station D, Ottawa, Ontario K1P 6P4, Canada, catalogue number CNMNC 87258.

How to cite: Gore, T. E. and McDonald, A. M.: Melansonite, IMA 2018-168, in: CNMNC Newsletter 52, Eur. J. Mineral., 32, https://doi.org/10.5194/ejm-32-1-2020, 2020.

\section{IMA no. 2019-052}

Ferrisanidine

$\mathrm{K}\left(\mathrm{Fe}^{3+} \mathrm{Si}_{3} \mathrm{O}_{8}\right)$

Arsenatnaya fumarole, second scoria cone of the Northern Breakthrough of the Great Tolbachik Fissure Eruption, Tolbachik volcano, Kamchatka Peninsula, Far Eastern region, Russia $\left(55^{\circ} 41^{\prime} \mathrm{N}, 160^{\circ} 14^{\prime} \mathrm{E}\right.$, 1200 m a.s.l.)

Nadezhda V. Shchipalkina, Igor V. Pekov, Sergey N. Britvin, Natalia N. Koshlyakova, Marina F. Vigasina, and Evgeny G. Sidorov

*E-mail: este158@yandex.ru
Feldspar group

Monoclinic: $C 2 / m$; structure determined

$a=8.678(4), \quad b=13.140(8), \quad c=7.335(5) \AA, \quad \beta=$ $116.35(8)^{\circ}$

4.283(52), 3.819(36), 3.529(30), 3.342(84), 3.285(100), 2.939(34), 2.643(30), 2.200(41)

Type material is deposited in the collections of the Fersman Mineralogical Museum, Russian Academy of Sciences, Leninskiy Prospekt 18-2, Moscow 119071, Russia, registration number 5414/1.

How to cite: Shchipalkina, N. V., Pekov, I. V., Britvin, S. N., Koshlyakova, N. N., Vigasina, M. F., and Sidorov, E. G.: Ferrisanidine, IMA 2019052, in: CNMNC Newsletter 52, Eur. J. Mineral., 32, https://doi.org/10.5194/ejm-32-1-2020, 2020.

\section{IMA no. 2019-053}

Fluorluanshiweiite

$\mathrm{KLiAl}_{1.5} \square_{0.5}\left(\mathrm{Si}_{3.5} \mathrm{Al}_{0.5}\right) \mathrm{O}_{10} \mathrm{~F}_{2}$

Nanyangshan deposit, North Qinling Orogen (NQO), western Henan Province, China $\left(110^{\circ} 43^{\prime} 55^{\prime \prime} \mathrm{E}\right.$, $\left.33^{\circ} 52^{\prime} 58^{\prime \prime} \mathrm{N}\right)$

Kai Qu*, Xianzhang Sima, Guowu Li, Guang Fan, Ganfu Shen, Xing Liu, Zhibin Xiao, Guo Hu, Linfei Qiu, and Yanjuan Wang

*E-mail: qukai_tcgs@foxmail.com

Mica group

Monoclinic: $C 2 / m$; structure determined

$a=5.2030(5), b=8.9894(6), c=10.1253(9) \AA, \beta=$ $100.68(1)^{\circ}$

8.427(25), 4.519(57), 4.121(25), 3.628(61), 3.349(60), 3.091(46), 2.586(100), 1.506(45)

Type material is deposited in the mineralogical collections of the Geological Museum of China, No. 16 Yangrou Hutong, Xisi, Beijing 100031, People's Republic of China, catalogue number M16085.

How to cite: Qu, K., Sima, X., Li, G., Fan, G., Shen, G., Liu, X., Xiao, Z., Hu, G., Qiu, L., and Wang, Y.: Fluorluanshiweiite, IMA 2019-053, in: CNMNC Newsletter 52, Eur. J. Mineral., 32, https://doi.org/10.5194/ejm-321-2020, 2020. 
IMA no. 2019-055

Thalliomelane

$\mathrm{TlMn}_{7.5}^{4+} \mathrm{Cu}_{0.5}^{2+} \mathrm{O}_{16}$

In a quarry at the Zalas village, near Cracow, Poland $\left(50^{\circ} 05^{\prime} 33^{\prime \prime} \mathrm{N}, 19^{\circ} 38^{\prime} 40^{\prime \prime} \mathrm{E}\right)$

Bożena Gołębiowska*, Adam Pieczka, Maciej Zubko, Andreas Voegelin, Jörg Göttlicher, and Grzegorz Rzepa

*E-mail: goleb@agh.edu.pl

Hollandite supergroup

Tetragonal: $I 4 / m$; structure determined

$a=9.866(1), c=2.8721(4) \AA$

3.120(85), 2.467(39), 2.407(66), 2.163(66), 1.838(45), $1.644(100), 1.545(39), 1.436(76)$

Type material is deposited in the collections of the Mineralogical Museum, University of Wrocław, Cybulskiego 30, 50-205 Wrocław, Poland, catalogue number MMWr IV8025.

How to cite: Gołębiowska, B., Pieczka, A., Zubko, M., Voegelin, A., Göttlicher, J., and Rzepa, G.: Thalliomelane, IMA 2019-055, in: CNMNC Newsletter 52, Eur. J. Mineral., 32, https://doi.org/10.5194/ejm-32-1-2020, 2020.

\section{IMA no. 2019-056}

Saccoite

$\mathrm{Ca}_{2} \mathrm{Mn}_{2}^{3+} \mathrm{F}(\mathrm{OH})_{8} \cdot 0.5\left(\mathrm{SO}_{4}\right)$

N'chwaning 3 Mine (NW section, upper part of the ore body), Kalahari Manganese Field, Northern Cape Province, South Africa $\left(27^{\circ} 07^{\prime} 45^{\prime \prime} \mathrm{S}, 22^{\circ} 51^{\prime} 56^{\prime \prime} \mathrm{E}\right)$

Gerald Giester*, Christian L. Lengauer, Dan Topa, Jens Gutzmer, and Karl-Ludwig Von Bezing

*E-mail: gerald.giester@univie.ac.at

New structure type

Tetragonal: $P 4 / n c c$; structure determined

$a=12.835(1), c=5.6213(6) \AA$

9.073(35), 4.537(95), 4.064(20), 3.010(100), 2.812(20),

$2.724(75), 1.975(35), 1.814(20)$

Type material is deposited in the mineralogical collections of the Naturhistorisches Museum Wien, Burgring 7, 1010 Vienna, Austria, inventory number O1784.
How to cite: Giester, G., Lengauer, C. L., Topa, D., Gutzmer, J., and Von Bezing, K.-L.: Saccoite, IMA 2019-056, in: CNMNC Newsletter 52, Eur. J. Mineral., 32, https://doi.org/10.5194/ejm-32-1-2020, 2020.

\section{IMA no. 2019-057}

Sergevanite

$\mathrm{Na}_{15}\left(\mathrm{Ca}_{3} \mathrm{Mn}_{3}\right)\left(\mathrm{Na}_{2} \mathrm{Fe}\right) \mathrm{Zr}_{3} \mathrm{Si}_{26} \mathrm{O}_{72}(\mathrm{OH})_{3} \cdot \mathrm{H}_{2} \mathrm{O}$

On a dump of the Karnasurt underground mine, Karnasurt mountain, Lovozero alkaline massif, Kola Peninsula, Murmansk Oblast, Russia $\left(67^{\circ} 53^{\prime} 25^{\prime \prime} \mathrm{N}\right.$, $\left.34^{\circ} 36^{\prime} 58^{\prime \prime} \mathrm{E}\right)$

Nikita V. Chukanov*, Ramiza K. Rastsvetaeva, Sergey M. Aksenov, Igor V. Pekov, Dmitry I. Belakovskiy, Svetlana A. Vozchikova, and Sergey N. Britvin

*E-mail: nikchukanov@yandex.ru

Eudialyte group

Trigonal: $R 3$; structure determined

$a=14.2179(1), c=30.3492(3) \AA$

7.12(70), 5.711(43), 4.321(72), 3.806(39), 3.551(39), 3.398(39), 2.978(95), 2.855(100)

Type material is deposited in the collections of the Fersman Mineralogical Museum, Russian Academy of Sciences, Leninskiy Prospekt 18-2, Moscow 119071, Russia, registration number 5423/1.

How to cite: Chukanov, N. V., Rastsvetaeva, R. K., Aksenov, S. M., Pekov, I. V., Belakovskiy, D. I., Vozchikova, S. A., and Britvin, S. N.: Sergevanite, IMA 2019-057, in: CNMNC Newsletter 52, Eur. J. Mineral., 32, https://doi.org/10.5194/ejm-32-1-2020, 2020.

\section{IMA no. 2019-058}

Polyarsite

$\mathrm{Na}_{7} \mathrm{CaMgCu}_{2}\left(\mathrm{AsO}_{4}\right)_{4} \mathrm{~F}_{2} \mathrm{Cl}$

Arsenatnaya fumarole, second scoria cone of the Northern Breakthrough of the Great Tolbachik Fissure Eruption, Tolbachik volcano, Kamchatka Peninsula, Far Eastern region, Russia $\left(55^{\circ} 41^{\prime} \mathrm{N}, 160^{\circ} 14^{\prime} \mathrm{E}\right.$, 1200 m a.s.1.)

Igor V. Pekov*, Natalia V. Zubkova, Atali A. Agakhanov, Dmitry I. Belakovskiy, Marina F. Vigasina, Sergey N. Britvin, Anna G. Turchkova, Evgeny G. Sidorov, and Dmitry Y. Pushcharovsky

*E-mail: igorpekov@mail.ru 
New structure type

Monoclinic: $I 2 / m$; structure determined

$a=8.4323(4), b=10.0974(4), c=10.7099(6) \AA, \beta=$ $90.822(4)^{\circ}$

5.357(98), 4.218(48), 3.304(49), 3.127(68), 2.783(61), 2.757(100), 2.704(54), 2.679(61)

Type material is deposited in the collections of the Fersman Mineralogical Museum, Russian Academy of Sciences, Leninskiy Prospekt 18-2, Moscow 119071, Russia, registration number 5417/1.

How to cite: Pekov, I. V., Zubkova, N. V., Agakhanov, A. A., Belakovskiy, D. I., Vigasina, M. F., Britvin, S. N., Turchkova, A. G., Sidorov, E. G., and Pushcharovsky, D. Y.: Polyarsite, IMA 2019-058, in: CNMNC Newsletter 52, Eur. J. Mineral., 32, https://doi.org/10.5194/ejm32-1-2020, 2020.

IMA no. 2019-059

Yurgensonite

$\mathrm{K}_{2} \mathrm{SnTiO}_{2}\left(\mathrm{AsO}_{4}\right)_{2}$

Arsenatnaya fumarole, second scoria cone of the Northern Breakthrough of the Great Tolbachik Fissure Eruption, Tolbachik volcano, Kamchatka Peninsula, Far Eastern region, Russia $\left(55^{\circ} 41^{\prime} \mathrm{N}, 160^{\circ} 14^{\prime} \mathrm{E}\right.$, 1200 m a.s.1.)

Igor V. Pekov*, Natalia V. Zubkova, Atali A. Agakhanov, Vasiliy O. Yapaskurt, Dmitry I. Belakovskiy, Marina F. Vigasina, Sergey N. Britvin, Anna G. Turchkova, Evgeny G. Sidorov, and Dmitry Y. Pushcharovsky

*E-mail: igorpekov@mail.ru

The Ti,Sn-ordered analogue of katiarsite

Orthorhombic: $P n a 2_{1}$; structure determined

$a=13.2681(6), b=6.6209(3), c=10.8113(5) \AA$

5.930(16), 5.656(100), 3.171(50), 3.082(11), 2.861(49), 2.830(82), 2.707(17), 2.600(14)

Type material is deposited in the collections of the Fersman Mineralogical Museum, Russian Academy of Sciences, Leninskiy Prospekt 18-2, Moscow 119071, Russia, registration number 5419/1.

How to cite: Pekov, I. V., Zubkova, N. V., Agakhanov, A. A., Yapaskurt, V. O., Belakovskiy, D. I., Vigasina, M. F., Britvin, S. N., Turchkova, A. G., Sidorov, E. G., and Pushcharovsky, D. Y.: Yurgensonite, IMA 2019059, in: CNMNC Newsletter 52, Eur. J. Mineral., 32, https://doi.org/10.5194/ejm-32-1-2020, 2020.
IMA no. 2019-060

Müllerite

$\mathrm{Pb}_{2} \mathrm{Fe}^{3+}\left(\mathrm{Te}^{6+} \mathrm{O}_{6}\right) \mathrm{Cl}$

Several workings on Otto Mountain, $2.5 \mathrm{~km}$ WNW of Baker, San Bernardino Co., California, USA: Aga mine $\left(35^{\circ} 16^{\prime} 19^{\prime \prime} \mathrm{N}, 116^{\circ} 05^{\prime} 42^{\prime \prime} \mathrm{W}\right)$, Aga Pit $\left(35^{\circ} 16^{\prime} 17^{\prime \prime} \mathrm{N}\right.$, $\left.116^{\circ} 05^{\prime} 39^{\prime \prime} \mathrm{W}\right)$, SW Cut $\left(35^{\circ} 16^{\prime} 20^{\prime \prime} \mathrm{N}, 116^{\circ} 06^{\prime} 04^{\prime \prime} \mathrm{W}\right)$, Bird Nest drift $\left(35^{\circ} 16^{\prime} 36^{\prime \prime} \mathrm{N}, 116^{\circ} 06^{\prime} 01^{\prime \prime} \mathrm{W}\right)$, Bird Nest E3 vein $\left(35^{\circ} 16^{\prime} 31^{\prime \prime} \mathrm{N}, 116^{\circ} 05^{\prime} 59^{\prime \prime} \mathrm{W}\right)$, and Library mine $\left(35^{\circ} 16^{\prime} 42^{\prime \prime} \mathrm{N}, 116^{\circ} 06^{\prime} 05^{\prime \prime} \mathrm{W}\right)$

Stuart J. Mills*, Anthony R. Kampf, Koichi Momma, Robert M. Housley, and Joseph Marty

*E-mail: smills@museum.vic.gov.au

The $\mathrm{Fe}^{3+}$ analogue of backite

Trigonal: $P 312$; structure determined

$a=5.2043(3), c=8.963(1) \AA$

4.495(40), 4.009(14), 3.174(100), 2.597(54), 2.247(20), 2.009(32), 1.697(25), 1.590(31)

Co-type material is deposited in the mineralogical collections of the Natural History Museum of Los Angeles County, 900 Exposition Boulevard, Los Angeles, California 90007, USA, catalogue numbers 73609 (SW Cut), 73610 (E3 vein), 73611 (Bird Nest drift) and 73612 (Aga mine), and the Museums Victoria, GPO Box 666, Melbourne 3001, Victoria, Australia, specimen number M54913 (Aga mine).

How to cite: Mills, S. J., Kampf, A. R., Momma, K., Housley, R. M., and Marty, J.: Müllerite, IMA 2019060, in: CNMNC Newsletter 52, Eur. J. Mineral., 32, https://doi.org/10.5194/ejm-32-1-2020, 2020.

\section{IMA no. 2019-061}

Drechslerite

$\mathrm{Tl}_{4}\left(\mathrm{Sb}_{4-\mathrm{x}} \mathrm{As}_{\mathrm{x}}\right) \mathrm{S}_{8}(1<x<2)$

Lengenbach quarry, Binn Valley, Valais, Switzerland $\left(46^{\circ} 21^{\prime} 54^{\prime \prime} \mathrm{N}, 8^{\circ} 13^{\prime} 15^{\prime \prime} \mathrm{E}\right)$

Dan Topa*, Stefan Graeser, Berthold Stoeger, Thomas Raber, and Chris Stanley

*E-mail: dan.topa@nhm-wien.ac.at

Related to lorándite and weissbergite

Triclinic: $P \overline{1}$; structure determined

$a=6.041(1), \quad b=6.166(1), \quad c=11.573(2) \AA, \quad \alpha=$ 100.89(3), $\beta=99.17(3), \gamma=104.54(3)^{\circ}$ 
3.613(89), 3.605(72), 3.495(81), 2.917(77), 2.898(100), 2.773(76), 2.296(50), 2.248(66)

Type material is deposited in the mineralogical collections of the Natural History Museum Vienna, Burgring 7, 1010 Vienna, Austria, specimen number O1181 (holotype), and the Natural History Museum Basel, Augustinergasse 2, 4051 Basel, Switzerland, specimen number S209 (co-type).

How to cite: Topa, D., Graeser, S., Stoeger, B., Raber, T., and Stanley, C.: Drechslerite, IMA 2019061, in: CNMNC Newsletter 52, Eur. J. Mineral., 32, https://doi.org/10.5194/ejm-32-1-2020, 2020.

\section{IMA no. 2019-062}

Michalskiite

$\mathrm{Fe}_{1.33}^{3+} \mathrm{Cu}_{2}^{2+}\left(\mathrm{MgFe}^{3+}\right)_{2}\left(\mathrm{VO}_{4}\right)_{6}$

In the dump of the Lichtenberg open pit, Ronneburg mining district, Thuringia, Germany $\left(50^{\circ} 51^{\prime} 20^{\prime \prime} \mathrm{N}\right.$, $\left.12^{\circ} 09^{\prime} 58^{\prime \prime} \mathrm{E}\right)$

Anthony R. Kampf*, Jakub Plášil, Radek Škoda, and Jiř́ Čejka

*E-mail: akampf@nhm.org

Isostructural with lyonsite

Orthorhombic: Pmcn; structure determined

$a=10.2356(9), b=17.369(2), c=4.9406(4) \AA$

$3.27(100), \quad 3.20(20), \quad 2.90(20), \quad 2.80(15), \quad 2.74(40)$,

2.52(50), 1.55(30), 1.42(25)

Type material is deposited in the mineralogical collections of the Natural History Museum of Los Angeles County, 900 Exposition Boulevard, Los Angeles, CA 90007, USA, catalogue number 67614.

How to cite: Kampf, A. R., Plášil, J., Škoda, R., and Čejka, J.: Michalskiite, IMA 2019-062, in: CNMNC Newsletter 52, Eur. J. Mineral., 32, https://doi.org/10.5194/ejm-32-1-2020, 2020.

\section{IMA no. 2019-064}

Evseevite

$\mathrm{Na}_{2} \mathrm{Mg}\left(\mathrm{AsO}_{4}\right) \mathrm{F}$

Arsenatnaya fumarole, second scoria cone of the Northern Breakthrough of the Great Tolbachik Fissure Eruption, Tolbachik volcano, Kamchatka Peninsula, Far Eastern region, Russia $\left(55^{\circ} 41^{\prime} \mathrm{N}, 160^{\circ} 14^{\prime} \mathrm{E}\right.$, 1200 m a.s.l.)
Igor V. Pekov*, Natalia V. Zubkova, Atali A Agakhanov, Dmitry I. Belakovskiy, Marina F. Vigasina, Vasiliy O. Yapaskurt, Sergey N. Britvin, Anna G. Turchkova, Evgeny G. Sidorov, and Dmitry Y. Pushcharovsky

*E-mail: igorpekov@mail.ru

The arsenate analogue of moraskoite

Orthorhombic: Pbcn; structure determined

$a=5.3223(1), b=14.1255(3), c=12.0047(3) \AA$

4.001(100), 3.527(31), 3.479(56), 3.041(45), 2.998(29), 2.657(44), 2.642(68), 2.613(36)

Type material is deposited in the collections of the Fersman Mineralogical Museum, Russian Academy of Sciences, Leninskiy Prospekt 18-2, Moscow 119071, Russia, registration number 5418/1.

How to cite: Pekov, I. V., Zubkova, N. V., Agakhanov, A. A., Belakovskiy, D. I., Vigasina, M. F., Yapaskurt, V. O., Britvin, S. N., Turchkova, A. G., Sidorov, E. G., and Pushcharovsky, D. Y.: Evseevite, IMA 2019064, in: CNMNC Newsletter 52, Eur. J. Mineral., 32, https://doi.org/10.5194/ejm-32-1-2020, 2020.

\section{IMA no. 2019-065}

Grokhovskyite

$\mathrm{CuCrS}_{2}$

Uakit iron meteorite (IIAB, $3.96 \mathrm{~kg}$ ), found in 2016 in the Baunt Evenk district, Republic of Buryatia, Russia $\left(55^{\circ} 29^{\prime} 47.50^{\prime \prime} \mathrm{N}, 113^{\circ} 33^{\prime} 47.98^{\prime \prime} \mathrm{E}\right)$

Victor V. Sharygin*, Grigoriy A. Yakovlev, Yurii V. Seryotkin, Nikolai S. Karmanov, Konstantin A. Novoselov, and Maxim S. Karabanalov

*E-mail: sharygin@igm.nsc.ru

Known synthetic analogue

Trigonal: $R 3 m$

$a=3.4794(8), c=18.702(4) \AA$

3.117(15), 2.975(100), 2.868(69), 2.533(44), 2.347(34), $1.999(70), 1.847(50), 1.740(67)$

Type material is deposited in the meteorite collections of the Central Siberian Geological Museum, V. S. Sobolev Institute of Geology and Mineralogy, Siberian Branch of the Russian Academy of Sciences, 3 prospekt Akad. Koptyuga, Novosibirsk 630090, Russia, registration number 52 (meteorite Uakit). 
How to cite: Sharygin, V. V., Yakovlev, G. A., Seryotkin, Y. V., Karmanov, N. S., Novoselov, K. A., and Karabanalov, M. S.: Grokhovskyite, IMA 2019065, in: CNMNC Newsletter 52, Eur. J. Mineral., 32, https://doi.org/10.5194/ejm-32-1-2020, 2020.

\section{IMA no. 2019-068}

Bennesherite

$\mathrm{Ba}_{2} \mathrm{Fe}^{2+} \mathrm{Si}_{2} \mathrm{O}_{7}$

In a paralava from Gurim Anticline, near Arad city, Hatrurim Complex (Mottled Zone), Negev desert, Israel $\left(31^{\circ} 12.5^{\prime} \mathrm{N}, 35^{\circ} 15.7^{\prime} \mathrm{E}\right)$

Arkadiusz Krzątała*, Biljana Krüger, Irina Galuskina, Yevgeny Vapnik, and Evgeny Galuskin

*E-mail: arkadiusz.krzatala@gmail.com

Melilite group

Tetragonal: $P \overline{4} 2_{1} m$; structure determined

$a=8.233(1), c=5.2854(8) \AA$

3.913(15), 3.682(13), 3.248(39), 3.021(100), 2.604(21), 2.147(22), 1.868(15), 1.855(18)

Type material is deposited in the collections of the Fersman Mineralogical Museum, Russian Academy of Sciences, Leninskiy Prospekt 18-2, Moscow 119071, Russia, registration number 5396/1.

How to cite: Krzątała, A., Krüger, B., Galuskina, I., Vapnik, Y., and Galuskin, E.: Bennesherite, IMA 2019068, in: CNMNC Newsletter 52, Eur. J. Mineral., 32, https://doi.org/10.5194/ejm-32-1-2020, 2020.

\section{IMA no. 2019-072}

Alcantarillaite

$\left[\mathrm{Fe}_{0.5}^{3+}\left(\mathrm{H}_{2} \mathrm{O}\right)_{4}\right]\left[\mathrm{CaAs}_{2}^{3+}\left(\mathrm{Fe}_{2.5}^{3+} \mathrm{W}_{0.5}^{6+}\right)\left(\mathrm{AsO}_{4}\right)_{2} \mathrm{O}_{7}\right]$

Alcantarilla mine, Belalcázar, Cordoba, Andalusia, Spain

Rupert Hochleitner, Christian Rewitzer, Ian E. Grey*, Alan Riboldi-Tunnicliffe, Anthony R. Kampf, Colin M. MacRae, W. Gus Mumme, Melanie Kaliwoda, Henrik Friis, and Carlos U. Martin

*E-mail: ian.grey@csiro.au

Structurally related to walentaite

Orthorhombic: Imma; structure determined

$a=24.038(8), b=7.444(3), c=10.387(3) \AA$
12.02(100), 6.343(23), 6.025(27), 4.257(17), 3.148(12), $3.016(38), 2.927(11)$

Type material is deposited in the collections of the Mineralogical State Collection, Theresienstrasse 41, 80333 Munich, Germany, collection number MSM37182 (holotype); the Natural History Museum, University of Oslo, P.O. Box 1172, Blindern, 0318 Oslo, Norway, collection number KNR44147 (holotype); and the Natural History Museum of Los Angeles County, 900 Exposition Boulevard, Los Angeles, CA 90007, USA, catalogue number 73568 (co-type).

How to cite: Hochleitner, R., Rewitzer, C., Grey, I. E., Riboldi-Tunnicliffe, A., Kampf, A. R., MacRae, C. M., Mumme, W. G., Kaliwoda, M., Friis, H., and Martin, C. U.: Alcantarillaite, IMA 2019-072, in: CNMNC Newsletter 52, Eur. J. Mineral., 32, https://doi.org/10.5194/ejm-32-1-2020, 2020.

\section{IMA no. 2018-117b}

Jingsuiite

$\mathrm{TiB}_{2}$

Luobusa ophiolite, ca. $200 \mathrm{~km}$ ESE of Lhasa, Tibet Autonomous Region, China $\left(29^{\circ} 11^{\prime} \mathrm{N}, 92^{\circ} 18^{\prime} \mathrm{E}\right.$, $5300 \mathrm{~m}$ a.s.1.)

Fahui Xiong, Xiangzhen Xu, Enrico Mugnaioli, Mauro Gemmi Richard Wirth, Edward S. Grew*, and Paul T. Robinson

*E-mail: esgrew@maine.edu

Known synthetic analogue

Hexagonal: $P 6 / \mathrm{mmm}$

$a=3.01(6), c=3.21(6) \AA$

3.218(21), 2.615(60), 2.029(100), 1.609(9), 1.510(20), $1.370(11), 1.211(12), 1.101(11)$

Type material is deposited in the mineralogical collections of the Chinese Geological Museum, No. 15 Xisiyangrou Hutong, Xicheng District, Beijing 100034, China, registration number M13816.

How to cite: Xiong, F., Xu, X., Mugnaioli, E., Gemmi, M., Wirth, R., Grew, E. S., and Robinson, P. T.: Jingsuiite, IMA 2018-117b, in: CNMNC Newsletter 52, Eur. J. Mineral., 32, https://doi.org/10.5194/ejm-32-1-2020, 2020. 


\section{New mineral proposals approved in November 2019}

\section{IMA no. 2019-034}

Bridgesite-(Ce)

$\mathrm{CaCe}_{2} \mathrm{Cu}_{6}\left(\mathrm{SO}_{4}\right)_{4}(\mathrm{OH})_{12} \cdot 8 \mathrm{H}_{2} \mathrm{O}$

Tynebottom Mine, Garrigill, Cumbria, UK $\left(54^{\circ} 46^{\prime} 16^{\prime \prime} \mathrm{N}, 2^{\circ} 24^{\prime} 26^{\prime \prime} \mathrm{W}\right)$

Mike S. Rumsey*, Frank Hawthorne, and John Spratt

*E-mail: m.rumsey@nhm.ac.uk

New structure type

Monoclinic: $C 2 / m$; structure determined

$a=24.801(5), \quad b=6.352(1), \quad c=11.245(2) \AA, \quad \beta=$ $114.51(3)^{\circ}$

6.376(99), 6.131(51), 4.116(55), 3.187(65), 2.822(52),

2.764(98), 2.698(94), 2.503(41)

Type material is deposited in the mineralogical collections of the Natural History Museum, Cromwell Road, London SW7 5BD, United Kingdom, registration numbers BM2007,81 (holotype), BM2007,82, and BM2007,83 (co-types).

How to cite: Rumsey, M. S., Hawthorne, F., and Spratt, J.: Bridgesite-(Ce), IMA 2019-034, in: CNMNC Newsletter 52, Eur. J. Mineral., 32, https://doi.org/10.5194/ejm-32-1-2020, 2020.

\section{IMA no. 2019-051}

Jahnsite-(NaMnMn)

$\mathrm{NaMn}^{2+}\left(\mathrm{Mn}^{2+} \mathrm{Fe}^{3+}\right)_{\Sigma 2} \mathrm{Fe}_{2}^{3+}\left(\mathrm{PO}_{4}\right)_{4}(\mathrm{OH})_{2} \cdot 8 \mathrm{H}_{2} \mathrm{O}$

Wiperaminga Hill West Quarry, Boolcoomatta Reserve, Olary Province, South Australia, Australia $\left(31^{\circ} 57^{\prime} 42^{\prime \prime} \mathrm{S}, 140^{\circ} 27^{\prime} 34^{\prime \prime} \mathrm{E}\right)$

Peter Elliott* and Anthony R. Kampf

*E-mail: peter.elliott@ adelaide.edu.au

Jahnsite group

Monoclinic: $P 2 / a$; structure determined

$a=15.136(1), b=7.2035(3), c=9.9876(6) \AA, \beta=$ $110.361(5)^{\circ}$

9.310(58), 5.035(26), 4.940(24), 4.664(25), 3.948(24), $3.551(34), 2.851(100), 2.608(27)$
Type material is deposited in the mineralogical collections of the South Australian Museum, North Terrace, Adelaide, South Australia 5000, Australia, registration number G34799.

How to cite: Elliott, P. and Kampf, A. R.: Jahnsite(NaMnMn), IMA 2019-051, in: CNMNC Newsletter 52, Eur. J. Mineral., 32, https://doi.org/10.5194/ejm-321-2020, 2020

\section{IMA no. 2019-054}

Chiyokoite

$\mathrm{Ca}_{3} \mathrm{Si}\left(\mathrm{CO}_{3}\right)\left\{\left[\mathrm{B}(\mathrm{OH})_{4}\right]_{0.5}\left(\mathrm{AsO}_{3}\right)_{0.5}\right\}(\mathrm{OH})_{6} \cdot 12 \mathrm{H}_{2} \mathrm{O}$

Fuka mine, Fuka, Bicchu-cho, Takahashi City, Okayama Prefecture, Japan $\left(34^{\circ} 46^{\prime} 00^{\prime \prime} \mathrm{N}\right.$, $133^{\circ} 25^{\prime} 59^{\prime \prime} \mathrm{E}$ )

Inna Lykova*, Nikita V. Chukanov, Igor V. Pekov, Vasiliy O. Yapaskurt, Leonid A. Pautov, Vladimir Y. Karpenko, Dmitry I. Belakovskiy, Dmitry A. Varlamov, Sergey N. Britvin, and Katharina S. Scheidl

*E-mail: ilykova@nature.ca

Ettringite group

Hexagonal: $P 6_{3}$; structure determined

$a=11.0119(5), c=10.5252(6) \AA$

9.53(100), 5.50(24), 4.618(11), 3.812(23), 3.412(15),

2.726(14), 2.521(19), 2.172(13)

Co-type material is deposited in the collections of the Fersman Mineralogical Museum, Russian Academy of Sciences, Leninskiy Prospekt 18-2, Moscow 119071, Russia, registration number 5412/1, and the Canadian Museum of Nature, 240 McLeod Street, Ottawa, ON K2P 2R1, Canada, catalogue number CNMNC 87294.

How to cite: Lykova, I., Chukanov, N. V., Pekov, I. V., Yapaskurt, V. O., Pautov, L. A., Karpenko, V. Y., Belakovskiy, D. I., Varlamov, D. A., Britvin, S. N., and Scheidl, K. S.: Chiyokoite, IMA 2019054, in: CNMNC Newsletter 52, Eur. J. Mineral., 32, https://doi.org/10.5194/ejm-32-1-2020, 2020.

\section{IMA no. 2019-073}

Jahnsite-(CaMnZn)

$\mathrm{CaMn}^{2+} \mathrm{Zn}_{2} \mathrm{Fe}_{2}^{3+}\left(\mathrm{PO}_{4}\right)_{4}(\mathrm{OH})_{2} \cdot 8 \mathrm{H}_{2} \mathrm{O}$

Pegmatite mine (67 m level), Hagendorf-Süd, Oberpfalz, Bavaria, Germany $\left(49^{\circ} 39^{\prime} 01^{\prime \prime} \mathrm{N}, 12^{\circ} 27^{\prime} 35^{\prime \prime} \mathrm{E}\right)$

Ian E. Grey*, Erich Keck, Anthony R. Kampf, Colin M. MacRae, John D. Cashion, and A. Matt Glenn 
*E-mail: ian.grey@csiro.au

Jahnsite group

Monoclinic: $P 2 / a$

$a=15.054(1), b=7.1890(6), c=10.030(2) \AA, \quad \beta=$ $111.273(7)^{\circ}$

9.356(40), 5.018(43), 4.677(32), 3.509(30), 3.473(24), 2.853(100), 1.961(22), 1.882(18)

Type material is deposited in the mineralogical collections of the Museums Victoria, P.O. Box 666, Melbourne, VIC 3001, Australia, registration number M55028 (holotype), and the Natural History Museum of Los Angeles County, 900 Exposition Boulevard, Los Angeles, CA 90007, USA, catalogue number 73927 (co-type).

How to cite: Grey, I. E., Keck, E., Kampf, A. R., MacRae, C. M., Cashion, J. D., and Glenn, M.: Jahnsite(CaMnZn), IMA 2019-073, in: CNMNC Newsletter 52, Eur. J. Mineral., 32, https://doi.org/10.5194/ejm-32-12020, 2020.

\section{IMA no. 2019-075}

Suenoite

$\square \mathrm{Mn}_{2} \mathrm{Mg}_{5} \mathrm{Si}_{8} \mathrm{O}_{22}(\mathrm{OH})_{2}$

Scortico Mn ore deposit, Fivizzano, Massa Carrara, Apuan Alps, Tuscany, Italy $\left(44^{\circ} 07^{\prime} 39^{\prime \prime} \mathrm{N}, 10^{\circ} 07^{\prime} 12^{\prime \prime} \mathrm{E}\right)$

Cristian Biagioni*, Marco Pasero, Elena Bonaccorsi, Ulf Hålenius, and Ferdinando Bosi

*E-mail: cristian.biagioni@unipi.it

Amphibole supergroup

Orthorhombic: Pnma; structure determined

$a=18.751(1), b=18.140(1), c=5.3173(3) \AA$

9.1(m), 8.4(s), 3.263(s), 3.085(vs), 2.851(m), 2.727(m), $2.615(\mathrm{~m}), 2.537(\mathrm{~m})$

Type material is deposited in the mineralogical collections of the Museo di Storia Naturale, Università di Pisa, Via Roma 79, Calci (Pisa), Italy, catalogue number 19891.

How to cite: Biagioni, C., Pasero, M., Bonaccorsi, E., Hålenius, U., and Bosi, F.: Suenoite, IMA 2019075, in: CNMNC Newsletter 52, Eur. J. Mineral., 32, https://doi.org/10.5194/ejm-32-1-2020, 2020.
IMA no. 2019-076

Badengzhuite

TiP

Cr-11 orebody, Luobusha ophiolite, ca. $200 \mathrm{~km}$ ESE of Lhasa, Tibet, China $\left(29^{\circ} 11^{\prime} \mathrm{N}, 92^{\circ} 18^{\prime} \mathrm{E}, 5300 \mathrm{~m}\right.$ a.s.1.)

Fahui Xiong, Xiangzhen Xu, Enrico Mugnaioli, Mauro Gemmi, Richard Wirth, Edward Grew*, and Paul T. Robinson

*E-mail: esgrew@maine.edu

Known synthetic analogue

Hexagonal: $P 6_{3} / m m c$; structure determined

$a=3.49(7), c=11.76(24) \AA$

3.022(14), 2.927(26), 2.688(31), 2.394(100), 2.107(33), $1.856(26), 1.745(44), 1.410(11)$

Type material is deposited in the mineralogical collections of the Chinese Geological Museum, Xisiyangrouhutong 15th, Xicheng District, Beijing, China, specimen number M13817.

How to cite: Xiong, F., Xu, X., Mugnaioli, E., Gemmi, M., Wirth, R., Grew, E., and Robinson, P. T.: Badengzhuite, IMA 2019-076, in: CNMNC Newsletter 52, Eur. J. Mineral., 32, https://doi.org/10.5194/ejm-321-2020, 2020.

IMA no. 2019-077

Zhiqinite

$\mathrm{TiSi}_{2}$

Cr-11 orebody, Luobusha ophiolite, ca. $200 \mathrm{~km}$ ESE of Lhasa, Tibet, China $\left(29^{\circ} 11^{\prime} \mathrm{N}, 92^{\circ} 18^{\prime} \mathrm{E}, 5300 \mathrm{~m}\right.$ a.s.1.)

Fahui Xiong, Xiangzhen Xu, Enrico Mugnaioli, Mauro Gemmi, Richard Wirth, Edward Grew*, and Paul T. Robinson

*E-mail: esgrew@maine.edu

Known synthetic analogue

Orthorhombic: $F d d d$; structure determined

$a=8.10(16), b=4.77(9), c=8.52(17) \AA$

3.702(9), 2.935(31), 2.265(100), 2.130(43), 2.081(78), 2.055(9), 1.810(39), 1.353(9)

Type material is deposited in the mineralogical collections of the Chinese Geological Museum, Xisiyangrouhutong 15th, Xicheng District, Beijing, China, specimen number M13817. 
How to cite: Xiong, F., Xu, X., Mugnaioli, E., Gemmi, M., Wirth, R., Grew, E., and Robinson, P. T.: Zhiqinite, IMA 2019-077, in: CNMNC Newsletter 52, Eur. J. Mineral., 32, https://doi.org/10.5194/ejm-32-1-2020, 2020.

\section{IMA no. 2019-079}

Bowlesite

PtSnS

Merensky Reef, Bushveld igneous complex, South Africa

Anna Vymazalová*, Federica Zaccarini, Giorgio Garuti, František Laufek, Daniela Mauro, Chris J. Stanley, and Cristian Biagioni

*E-mail: anna.vymazalova@geology.cz

Isotypic with cobaltite

Orthorhombic: $P c a 2_{1}$; structure determined

$a=6.12, b=6.12, c=6.10 \AA$

3.526(40), 3.054(47), 2.490(36), 2.159(82), 1.843(100), $1.401(64), 1.368(32), 1.367(35)$

Type material is deposited in the mineralogical collections of the Department of Earth Sciences, Natural History Museum, London SW7 5BD, UK, catalogue number BM2019,11 (holotype), and the Museo di Storia Naturale, Università di Pisa, Via Roma 79, Calci (Pisa), Italy, catalogue number 19909 (anthropotype).

How to cite: Vymazalová, A., Zaccarini, F., Garuti, G., Laufek, F., Mauro, D., Stanley, C. J., and Biagioni, C.: Bowlesite, IMA 2019-079, in: CNMNC Newsletter 52, Eur. J. Mineral., 32, https://doi.org/10.5194/ejm-32-12020, 2020.

\section{IMA no. 2019-080}

Gorerite

$\mathrm{CaAlFe}_{11}^{3+} \mathrm{O}_{19}$

"Olive unit", Hatrurim Complex, Negev desert, near Arad city, Israel $\left(31^{\circ} 13^{\prime} 58^{\prime \prime} \mathrm{N}, 35^{\circ} 16^{\prime} 02^{\prime \prime} \mathrm{E}\right)$

Evgeny V. Galuskin, Biljana Krüger, Irina O. Galuskina, Hannes Krüger, Krzysztof Nejbert, Yevgeny Vapnik, and Takashi Tomizaki

*E-mail: evgeny.galuskin@us.edu.pl

Magnetoplumbite group

Hexagonal: $P 6_{3} / m m c$; structure determined $a=5.8532(4), c=22.7730(2) \AA$

2.795(40), 2.695(100), 2.508(94), 2.306(39), 2.136(27),

1.594(31), 1.571(46), 1.398(42)

Type material is deposited in the collections of the Fersman Mineralogical Museum, Russian Academy of Sciences, Leninskiy Prospekt 18-2, Moscow 119071, Russia, registration number 5420/1.

How to cite: Galuskin, E. V., Krüger, B., Galuskina, I. O., Krüger, H., Nejbert, K., Vapnik, Y., and Tomizaki, T.: Gorerite, IMA 2019-080, in: CNMNC Newsletter 52, Eur. J. Mineral., 32, https://doi.org/10.5194/ejm-321-2020, 2020.

\section{IMA no. 2019-081}

Alexkuznetsovite-(La)

$\mathrm{La}_{2} \mathrm{Mn}\left(\mathrm{CO}_{3}\right)\left(\mathrm{Si}_{2} \mathrm{O}_{7}\right)$

Mochalin Log REE deposit, $14 \mathrm{~km} \mathrm{~N}$ of the city of Kyshtym, Chelyabinsk Oblast', southern Ural Mountains, Russia ( $\left.55^{\circ} 48^{\prime} 42^{\prime \prime} \mathrm{N}, 60^{\circ} 33^{\prime} 46^{\prime \prime} \mathrm{E}\right)$

Anatoly V. Kasatkin*, Natalia V. Zubkova, Igor V. Pekov, Nikita V. Chukanov, Radek Škoda, Atali A. Agakhanov, Dmitriy I. Belakovskiy, and Dmitry Y. Pushcharovsky

*E-mail: anatoly.kasatkin@gmail.com

The La- and Mn-dominant analogue of biraite-(Ce)

Monoclinic: $P 2{ }_{1} / c$; structure determined

$a=6.5642(3), b=6.7689(3), c=18.721(1) \AA, \beta=$ $108.684(6)^{\circ}$

4.595(63), 4.489(56), 4.331(62), 4.208(50), 3.171(49), 2.962(100), 2.785(76), 2.692(59)

Type material is deposited in the collections of the Fersman Mineralogical Museum, Russian Academy of Sciences, Leninskiy Prospekt 18-2, Moscow 119071, Russia, registration number 5416/1.

How to cite: Kasatkin, A. V., Zubkova, N. V., Pekov, I. V., Chukanov, N. V., Škoda, R., Agakhanov, A. A., Belakovskiy, D. I., and Pushcharovsky, D. Y.: Alexkuznetsovite-(La), IMA 2019-081, in: CNMNC Newsletter 52, Eur. J. Mineral., 32, https://doi.org/10.5194/ejm-32-1-2020, 2020. 
IMA no. 2018-125a

Fehrite

$\mathrm{MgCu}_{4}\left(\mathrm{SO}_{4}\right)_{2}(\mathrm{OH})_{6} \cdot 6 \mathrm{H}_{2} \mathrm{O}$

Mina Casualidad, near Baños de Alhamilla, Almeria, Spain $\left(36^{\circ} 57^{\prime} 45.53^{\prime \prime} \mathrm{N}, 2^{\circ} 23^{\prime} 44.17^{\prime \prime} \mathrm{W}\right)$

Jochen Schlüter*, Thomas Malcherek, Christian Rewitzer, Rupert Hochleitner, Dirk Müller, and Amanda Günther

*E-mail: jochen.schlueter@uni-hamburg.de

The Mg analogue of ktenasite

Monoclinic: $P 22_{1} / c$; structure determined

$a=5.6062(8), \quad b=6.129(1), \quad c=23.834(3) \AA, \quad \beta=$ $95.29(1)^{\circ}$

11.936(100), $\quad 5.920(31), \quad 4.845(11), \quad 3.933(11)$, 2.964(10), 2.775(9), 2.659(12), 2.552(8)

Type material is deposited in the collections of the Mineralogical Museum, Centrum für Naturkunde, University Hamburg, Grindelallee 48, 20146 Hamburg, Germany, catalogue no. MM1544 (holotype), and the Mineralogische Staatssammlung München, Theresienstrasse 41, 80333 Munich, Germany, catalogue no. MSM36473 (co-type).

How to cite: Schlüter, J., Malcherek, T., Rewitzer, C., Hochleitner, R., Müller, D., and Günther, A.: Fehrite, IMA 2018-125a, in: CNMNC Newsletter 52, Eur. J. Mineral., 32, https://doi.org/10.5194/ejm-32-1-2020, 2020.

IMA no. 2018-149a

Petrovite

$\mathrm{Na}_{8}(\mathrm{NaCu}) \mathrm{Cu}_{2} \mathrm{Na}\left(\mathrm{SO}_{4}\right)_{8}$

Second scoria cone of the Northern Breakthrough of the Great Tolbachik fissure eruption, Tolbachik volcano, Kamchatka, Far Eastern region, Russia $\left(55^{\circ} 41^{\prime} \mathrm{N}\right.$, $160^{\circ} 14^{\prime} \mathrm{E}, 1200 \mathrm{~m}$ a.s.l.)

Andrey P. Shablinskii, Lidiya P. Vergasova, Stanislav K. Filatov*, and Svetlana V. Moskaleva

*E-mail: filatov.stanislav@ gmail.com

Chemically similar to saranchinaite

Monoclinic: $P 22_{1} / c$; structure determined

$a=12.615(2), \quad b=9.026(1), \quad c=12.717(2) \AA, \quad \beta=$ $108.311(3)^{\circ}$
11.59(61), $7.27(38), \quad 4.54(33), \quad 3.88(75), \quad 3.68(52)$, 3.11(36), 2.623(100), 1.931(35)

Type material is deposited in the collections of the Mineralogical Museum of the Saint Petersburg State University, University Emb. 7/9, St. Petersburg 199034, Russia, catalogue number 1/19696.

How to cite: Shablinskii, A. P., Vergasova, L. P., Filatov, S. K., and Moskaleva, S. V.: Petrovite, IMA 2018149a, in: CNMNC Newsletter 52, Eur. J. Mineral., 32, https://doi.org/10.5194/ejm-32-1-2020, 2020.

\section{Nomenclature/classification proposals approved in October 2019}

\section{IMA 19-E: revalidation of dienerite}

Proposal 19-E is accepted, and the mineral dienerite is revalidated. This species shows an ideal chemical formula $\mathrm{Ni}_{3} \mathrm{As}$, with $a=9.6206(9) \AA$, space group $I \overline{4} 3 d$. It is isotypic with domeykite, $\mathrm{Cu}_{3} \mathrm{As}$, and the neotype sample is deposited in the collections of the Museo di Storia Naturale, Università di Firenze, Italy, under the catalogue number 3364/I.

\section{IMA 19-F: redefinition of coquimbite}

Proposal 19-F is accepted, and coquimbite is redefined as $\mathrm{AlFe}_{3}\left(\mathrm{SO}_{4}\right)_{6}\left(\mathrm{H}_{2} \mathrm{O}\right)_{12} \cdot 6 \mathrm{H}_{2} \mathrm{O}(\mathrm{Z}=2)$. Neotype material is the sample studied by Fang and Robinson (1970) from the Tierra Amarilla (the original type locality described by Rose, 1833) and kept in the US National Museum, Washington DC, USA, catalogue number 12548. In order to be compared with coquimbite, the chemical formulae of paracoquimbite and aluminocoquimbite are also redefined as $\mathrm{Fe}_{4}\left(\mathrm{SO}_{4}\right)_{6}\left(\mathrm{H}_{2} \mathrm{O}\right)_{12} \cdot 6 \mathrm{H}_{2} \mathrm{O}(\mathrm{Z}=6)$ and $\mathrm{Al}_{2} \mathrm{Fe}_{2}\left(\mathrm{SO}_{4}\right)_{6}\left(\mathrm{H}_{2} \mathrm{O}\right)_{12} \cdot 6 \mathrm{H}_{2} \mathrm{O}(\mathrm{Z}=2)$, respectively. Coquimbite and paracoquimbite are characterized by homeotypic structures, so they constitute the "coquimbite group". Aluminocoquimbite, characterized by a different topology, does not belong to the coquimbite group.

\section{Nomenclature/classification proposals approved in November 2019}

\section{Revision of the ideal formula of ktenasite}

After the approval of fehrite, $\mathrm{MgCu}_{4}\left(\mathrm{SO}_{4}\right)_{2}(\mathrm{OH})_{6} \cdot 6 \mathrm{H}_{2} \mathrm{O}$ (IMA no. 2018-125a; see above), the ideal formula of the related mineral ktenasite is modified from $(\mathrm{Cu}, \mathrm{Zn})_{5}\left(\mathrm{SO}_{4}\right)_{2}(\mathrm{OH})_{6} \cdot 6 \mathrm{H}_{2} \mathrm{O}$ to $\mathrm{ZnCu}_{4}\left(\mathrm{SO}_{4}\right)_{2}(\mathrm{OH})_{6} \cdot 6 \mathrm{H}_{2} \mathrm{O}$. This is an executive decision made by the CNMNC. 


\section{Revision of the ideal formula of leisingite}

The mineral leisingite has always been assumed to have the chemical formula $\mathrm{CuMg}_{2} \mathrm{Te}^{6+} \mathrm{O}_{6} \cdot 6 \mathrm{H}_{2} \mathrm{O}$. A careful reading of the papers with the description of the new mineral and of its crystal structure [Mineral. Mag. 60 (1996), 653-657; Can. Mineral. 35 (1997), 759-763] made it clear that $\mathrm{Cu}$ is by far the dominant cation at the $2 c$ site, whereas $\mathrm{Mg}$ is the dominant cation at the $1 b$ site. Therefore the formula must be changed to $\mathrm{Cu}_{2} \mathrm{MgTe}^{6+} \mathrm{O}_{6} \cdot 6 \mathrm{H}_{2} \mathrm{O}$. Thanks to Owen Missen for spotting it. 